\title{
Melanosis Neurocutánea Familiar
}

\author{
Drs. Maria Eugenia Willshaw L. ${ }^{(1)}$, Manuel Pérez F. ${ }^{(2)}$ e Yves Lcassie $\mathbf{S}{ }^{(3)}$
}

\section{Familial Neurocutaneous Melanosis}

\begin{abstract}
Neurocutaneous melanosis is a severe melanocy tic hamartomatosis of skin and pia-arachnoid. Most of the reported cases have been sporadic, with no evidence of mendelian inheritance.

We report a 13 years old girl with the impressive abnormalities of this anomalad besides scoliosis and a diffuse euthyroid goiter. The finding of pigmented nevi in hes mother and other family members, allow to suggest an autosomal dominant inheritance, with variable expressivity, for this condition. The ocurrence of scoliosis and goiter in some sibs and their relationship with the neurocutaneous melanosis is dixcussed.
\end{abstract}

\section{INTRODUCCION}

La Melanosis Neurocutánea (MN) fue descrita por primera vez en 1861 por Rokitansky en una niña de 14 años con un nevus gigante benigno de la piel, hidrocefalia, retardo mental e infiltración pigmentarja de la piamadre en la autopsia (1). En 1948, Van Bogaert la reconoce como una entidad y la denomina "Melanosis Neurocutánet" (2). En ta actualidad es considerada una anomalía siendo incluida en el grupo de las hamartosis. (3).

El diagnóstico de esta afección es clínico y se basa en manifestaciones:

Cutáneas: Nevus pigmertados gigantes ubicados preferentemente en tronco y abdomen (en traje de baño), a veces pilosos, acompañados de múltiples nevus pequeños, en número variable, habiéndose descrito hasta 190 elementos en un solo paciente. Histológicamente son benignos y sin riesgo de malignización. Las lesiones cutáneas permiten el diagnóstico presuntivo desde el naçimiento.

Neurológicas: Compromiso precoz, caracterizado por convulsiones rebeldes al tratamiento, con deterioro progresivo e hidrocefalia. Estas manifestaciones se atribuyen al depósito pigmentario en leptomeninges, evidentes en el estudio histopatológico. Este es el criterio diagnóstico confirmatorio, ya que mientras no se encuentre el depósito

1 Servicio de Endocrinología Intantil, Hospital Milikar.

2 Servicio de Neurología, Hospital Militar.

3 Unidad de Genética, INTA, Universidad de Chile. melánico en el SNC, el diagnóstico es sôlo presuntivo. Estas lesiones tienen un alto riesgo de malignización (4).

Esta afección pueđe coexistir e interrelacionarse con otras hamartosis. Al igual que otras enfermedades de este grupo, es posible que existan tantbién formas incompletas (4). Su diagnóstico exacto es importante no sólo por el consejo genético, sino también por su tendencia a malignizar.

El pronóstico de esta afección es malo, dado que la mayoría de los pacientes fallecen antes de los 2 atios de edad y sólo $1 / 6$ de los casos supera $\operatorname{los} 25$ años y con un deterioro progresivo del SNC (3).

Afortunadamente esta es una afección raca y generalmente esporádica. Hemos encontrado solamente 2 publicaciones de $\mathrm{MN}$ familiar $(2,5)$.

El estudio de una paciente con el cuadro característico de $\mathrm{MN}$ y el hallazgo de manifestaciones cutáneas en otros familiares, sugiriendo una herencia mendeliana en esta familia, han motivado esta publicación.

\section{CASO CLINICO}

(Obs. 92257). Paciente de sexo femenino, de 13 años de edad, hija de padres sanos no consanguíneos, sin antecedentes familiares de importancia. 
producto de un embarazo y parto normales ( $\mathrm{PN}=$ 3800 gramos), que desde el nacimiento presenta intensas y extensas lesiones melänicas en tronco y parte alta del abdomen (en traje de baño), con numerosísimos nevi pigmentados de tamaño variable, algunos pilosos, distribuidos en todo e] cuerpo. A los 4 meses de edad, presenta convulsiones tónico-clónicas generalizadas, que persisten hasta la actualidad, a pesar de tratamiento a permanencia con diversos anticonvulsivantes.

En el examen físico, además de las lesiones cutáneas descritas (Fig. 1), presentaba escoliosis dorsoiumbar discreta, bocio difuso grado II, retraso estatural (talla $141 \mathrm{~cm}$ ) y obesidad (43 kg.).

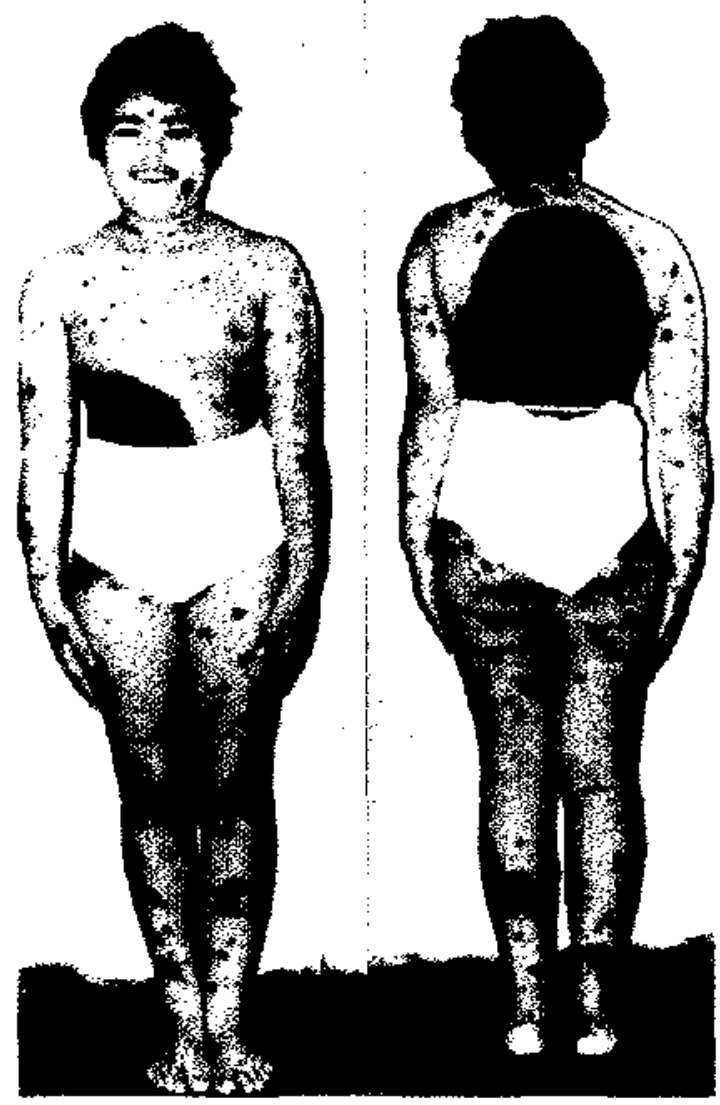

Figura 1

Su desarrollo puberal correspondía a etapa II] de Tanner. Una evaluación intelectual demostró un C. I. global de 69 .

Los exámenes de laboratorio realizados se presentan en la Tabla 1.
Tabla 1

Exámenes de Laboratorio

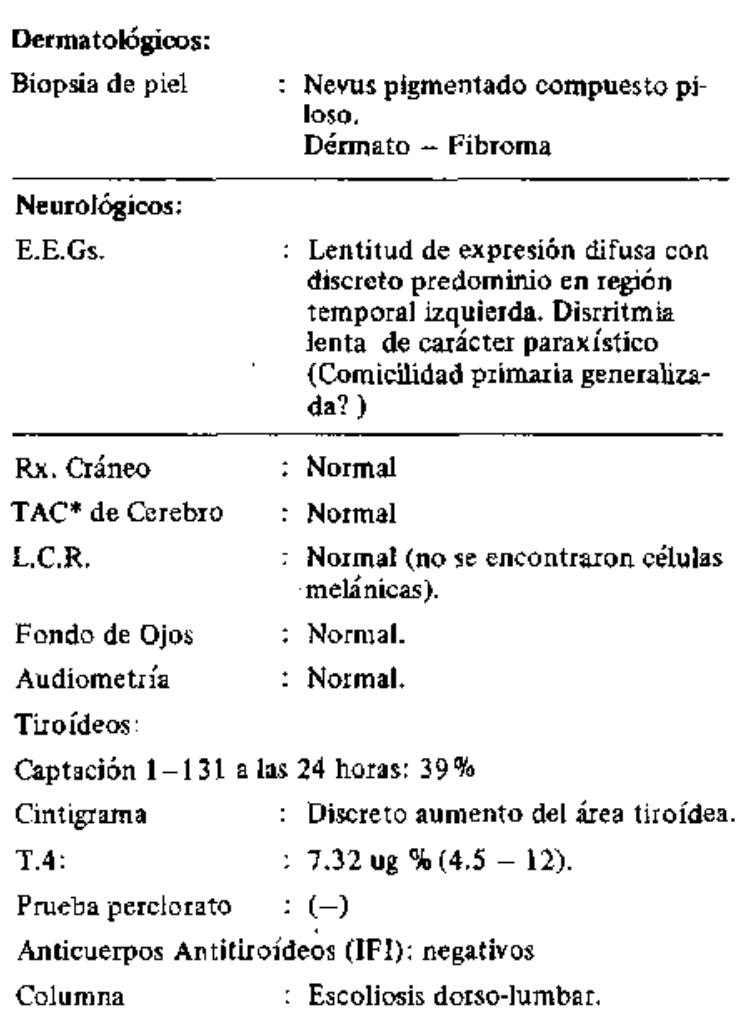

\section{Estudio Familiar:}

El examen del grupo familiar (Fig. 2), demostró normalidad en el padre $\left(I_{1}\right)$, aunque dos de sus cinco hermanos presentarian pequeños nevus (no pudieron ser examinados). La madre $\left(\mathbf{I}_{2}\right)$ presentaba cuatro nevi pigmentados en cara anterior $y$ posterior del tórax, acompañados de varios otros de tamaño reducido. De los seis hermanos de la probando, una ( $\left.\mathrm{II}_{3}\right)$ presentaba nevus, bocio difuso de grado Il y escoliosis (Fig. 3), otra ( $\mathrm{II}_{5}$ ) bocio y escoliosis, dos $\left(\mathrm{II}_{2} \mathrm{y}_{6}\right)$ sólo bocio y dos (II, y 7) no presentaban nínguna manifestación. El estudio dermatoglífico reveló una tendencia familiar al aumento de círculos en los dedos, presillas con bolsillo central en los meñiques (CPL) y trirradios axiales distales en algunos miembros de la familia.

El estudio del bocio en los hermanos demostró eutiroidismo, sin descarga de yodo al dar perclorato. Todos tenian audición normal.

-TAC = Tomografio Axial Computada 


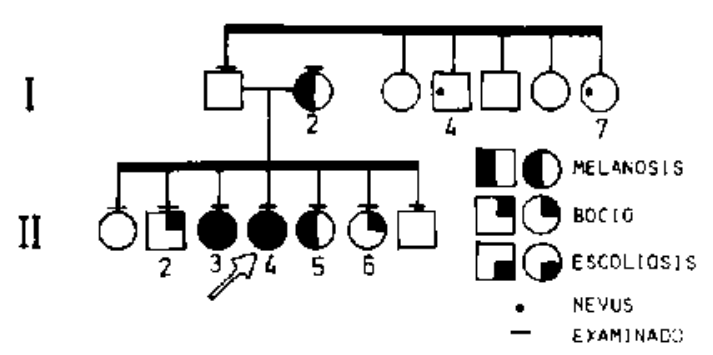

Figura 2

\section{DISCUSION}

Aunque el diagnóstico confimatorio de $\mathrm{MN}$ requiere la comprobación de depósito pigmentario en leptomeninges (4), creemos que nuestra probando presenta hs características clínicas fundamentales de esta afección. El hallazgo de iesiones cutáneas congénitas, benignas, características en la biopsia de piel, asociadas a un cuadro convulsivo precoz y rebelde at tratamiento, con un evidente $y$ progresivo deterioro mental, permiten establecer este diagnóstico (3). Una evidencia indirecta del compromiso de leptomeninges sería la presencia de célutas melánicas en el LCR $(3,4)$, en nuestro caso fue negativa.

E] origen embrionario de los melanocitos. a partir de la cresta neural, explica la relación entre melanosis cutánea y del SNC (4).

Las características clínicas del bocio y Jos exámenes de laboratorio en la probando y sus hermanas no corresponden a las descritas en los bocios familiares. que generalmente son de gran tamaño, con defecto de organificación y acompañados de otras manifestaciones como sordera. Tampoco se encontró evidencias de tiroiditis u otras causas habituales de bocio. La concomitancia de bocio y escoliosis $\left(\mathrm{II}_{3} y_{4}\right)$ con las lesiones cutáneas, podrian ser coincidencia.

Sin embargo, otras enfermedades neurocutáneas suelen acompañarse de escoliosis, sugiriendo que ésta podría ser un elemento más en esta anomalia. No tenemos una explicación adecuada para relacionar etiológicamente el bocio con la $\mathrm{MN}$. a menenos que fuese un efecto pleiotrópico del gen.

El análisis de esta genealogía es sugerente, de herencia autosomica dominante con expresividad variable. Sin embargo, una herencia poligénica también podría explicar los hallazgos en esta familia.

En la revisión de la literatura efectuada, solamente encontramos dos publicaciones de MN familiar $(2,5)$. Lo raro de esta afección, las manifestaciones concomitantes que podrían ser parte de este sindrome y su presentación excepcionalmente familiar, justifican su publicación.

\section{RESUMEN}

La Melanosis Neurocutánea (MN) es una hamartosis melanocítica de piel y pia-aracnoides grave, caracterizada clinicamente por múltiples nevus pigmentados congénitos, preferentemente en tronco y abdomen. convulsiones y deterioro progresivo del SNC. Esta asociación se deberia a una displasia congénita de la cresta neural. La mayoría de los casos descritos han sido esporídicos, sin que existan evidencias ciertas de herencia mendeliana.

Se presenta una paciente de 13 años de edad, con intensas lesiones melinicas de piel, historia de convulsiones rebeldes desde los 4 meses de vida $y$ retardo mental moderado. Al examen físico se encontró, además, escoliosis y bocio difuso eutjroídeo. El examen familias demost tó la presencia de pequeños nevus pigmentados en la madre y una hermana, la cual también presentaba bocio $y$ escoliosis. Estas dos manifestaciones estaban presentes en otra hermana, mientras que otros dos hermanos tenian exclusivamente la alteración tiroidea.

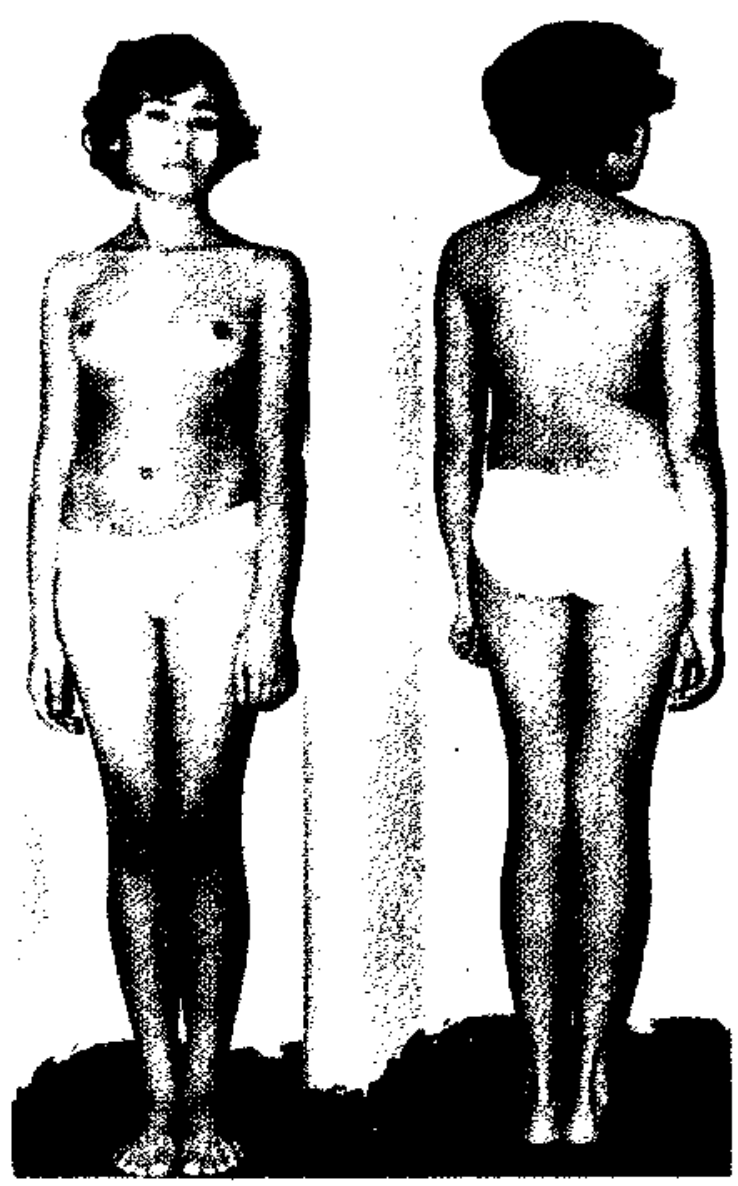

Figura 3 
Aunque 2 tios paternos presentarian pequeños nevus pigmentados, esta genealogía permite postular una herencia dominante con expresividad variable para la $\mathrm{MN}$. Se discute la relación de $\mathrm{MN}$ con bocio $y$ escoliosis en esta familia.

\section{AGRADECIMIENTOS}

Se agradece la colaboración del Servicio de Dermatología del Hospital J. J. Aguirre, donde se efectuó la biopsia de piel y los anticuerpos antitiroídeos, asi como la experta labor secretarial de la Sra. Viola Lyon.

\section{REFERENCIAS}

${ }^{1}$ Rokitansky, G.: Ein Ausgezeichneter fall von PigmentMal mit ausge breiteter pigmentirung der inneren hirn - und Ruckenmark Shäute. Allg. Wien med ZTG 6: 113, 1861 .

2 Van Bogaert, L.: La Mélanose Neurocutanée Diffuse Héredofamiliale. Bull. Acad. R. Med. Belg. 13:397, 1948.

${ }^{3}$ Smith, D. W.: Recognizable Patterns of Human Melformation. W.B. Saunders Company, Philadelphia, Second Fidition, 1976.

4 Fox, H,; Emery, J.L.; Goodbody. R.A.; Yates, P.O.: Ncurocutaneous Melanosis. Arch. Dis. Child. 39: 508, 1964.

5 Fischer, $S$.: Primary perivascular cerebral, cerebellar and leptomenungeal melanoma (congenital aphasia and familial predisposition to nacvi verrucosiy. Acta Psychiat. Scand. 31:21, 1956. 